\title{
Multi-criteria analysis of wearable activity- fitness trackers and 3D concept models of smart jewellery
}

\author{
Raluca Marinescu (Repanovici)* \\ Transylvania University of Braşov, Department of Engineering and Industrial Management, Mihai \\ Viteazul 5, Braşov, Romania
}

\begin{abstract}
The technological development of wearable activity tracking devices with a whole range of functions is considered in this paper. Experts from different decision-making groups performed the multiple attribute evaluation of three devices for heart rate, exercise and sleep monitoring: a watch, a bracelet and a ring. MCA is applied to determine the relative weights of evaluation criteria. The result shows that the ring is the most suitable tracker that fits seamlessly into everyday life. Following the result, several 3D models of smart jewellery are presented for rapid prototyping.
\end{abstract}

\section{Introduction}

Smartphones and activity-fitness trackers are essential devices in our everyday life and due to their power and efficiency, more and more people are using wearable trackers and mobile health applications to track their medical condition and to measure physical activity and sleep [1]. The wearable trackers are becoming progressively popular in personal healthcare and the various choices in the market are increasing.

With customers searching for products that best suit their personal needs, as Mike Strasser, cofounder and CEO of Motiv Inc. noticed [2]: "despite how fast the wearable market is growing, over half of those who buy a fitness tracker lose interest and discontinue use because current solutions are uncomfortable, unattractive and don't provide truly meaningful insights", the companies started to develop smarter and more user-friendly devices.

The selection of optimal solutions regarding the wearable activity tracking devices is a complex process as it depends on several criteria. Multi-Criteria Analysis (MCA) can be used to identify a single most preferred alternative, to rank different solutions, or simply to distinguish acceptable from unacceptable possibilities. MCA establishes preferences between alternatives by reference to an explicit set of objectives that the decision-making experts have identified, and for which it has established measurable criteria to assess the extent to which the objectives have been achieved [3]. The main stages of the MCA are the following: establishing the decision context, identifying the alternatives, identifying the

\footnotetext{
*Corresponding author: raluca-maria.marinescu@unitbv.ro
} 
criteria, assigning criteria weights, construction of the evaluation matrix, assessing performance levels and ranking the alternatives [3, 4].

Rapid prototyping is the result of $3 \mathrm{D}$ printing process. Based on layered manufacturing, 3D printing is designed for Computer Aided Design (CAD) software such as Autodesk Inventor or SolidWorks, where the model is created. After the creation phase is completed, the model is exported with a readable format and sent to the printer system. The $3 \mathrm{D}$ printer creates objects in layers, building from the bottom up. Saving money and time, this new technology has set new rules to the manufacturing process and its usage for both commercial and non-commercial activities will increase rapidly [5].

\section{Identifying the alternatives}

Based on the technological development, three alternatives of wearable trackers, introduced in Wearable Technologies category at Consumer Electronics Show (CES) 2017 in Las Vegas, are considered, and the features of each alternative are described in this paper [6].

\subsection{Alternative solutions}

\subsubsection{Inbody watch}

Awarded with 'Innovations Award Honoree' at CES 2017 [6], the InBody Watch is a wearable tracker capable to measure heart rate, body composition as well as track stress levels. InBody Watch helps people to manage their heart health and risk of developing cardiovascular diseases by reporting Pulse Wave Velocity. Using Bioelectrical Impedance Analysis, the InBody Watch provides body composition analysis, calculates the stress levels via Heart Rate Variability, measures continuous heart rate, and maintains the other fitness tracking features such as step counting, distance travelled, calories burned, activity and sleep tracking. In addition, it uses a precision motion sensor and a powerful algorithm that automatically recognize 12 workout motions to create personalized exercise program. The InBody Watch also comes equipped with smart features such as touch screen, autotime, date and battery life indicators, notifications for calls and texts, and a dedicated BodyKey mobile application to provide measurement analysis [7].

\subsubsection{Fitbit charge 2}

The Fitbit Charge 2, awarded with 2017 'Innovation Award' at CES 2017 [6], is a heart rate and fitness wristband equipped with a continuous heart rate monitor, activity and sleep tracker, connected GPS and other revolutionary features. Packed with a PurePulseTM heart rate monitor, Fitbit Charge 2 measures heart rate, provides simplified heart rate zones and resting heart rate trends, monitors burned calories and cardio fitness level. As all-day activity trackers, Fitbit Charge 2 tracks steps, distance walked, floors climbed, active minutes and sleep patterns, but with its SmartTrackTM technology, the bracelet automatically recognizes select exercises (running, biking and more) and records them in the Fitbit app. By tracking real-time heart-rate, Fitbit Charge 2 can provide guided breathing sessions with personalised prompts. All these measurements, together with calls, texts and calendar alerts, are displayed on the Fitbit Charge 2 OLED display. The dedicated Fitbit mobile application is the bigger picture of the screen, showing easy-to-read charts and graphs to visualize the progress, but also to provide personalized prompts [8]. 


\subsubsection{Motiv ring}

Awarded with 'Best of Innovation' at CES 2017 [6], this ring (Motiv Ring), is a unique device that changes the way people measure activity and sleep. Essentially, Motiv Ring is the first highly advanced wearable device, shaped as a ring, capable to measure hear rate, sleep patterns and daily activity through day and night. As a simple fitness tracker, the most important thing about the Motiv Ring is the discreet and elegant design that fits seamlessly into everyday life. Allowing to effortlessly measure activity and sleep, the ring is comfortable enough to be worn at all times, in any situation, even in the shower. Built from high-quality ultralight Titanium, this piece of smart jewellery offers a general layout of activity and health. With the most advanced technology, the device is waterproof and armed with a smart LED sensor, optical heart rate sensor and a 3-axis accelerometer. All the information measured by Motiv Ring is synced with the dedicated mobile application Motiv App. The ring is available in two different colours and comes in seven sizes [9].

\subsection{Comparison of the alternative solutions}

The comparison of the three wearable activity-fitness trackers is presented in Table 1.

Table 1. Comparison of the Alternative Wearable Activity-Fitness Trackers [7, 8, 9].

\begin{tabular}{|c|c|c|c|}
\hline Features & InBody WATCH & Fitbit Charge 2 & Motiv Ring \\
\hline Design & Timeless & Pleasing & Elegant and discreet \\
\hline Waterproof & 1 meter & 3ATM (30 meters) & 5ATM (50 meters) \\
\hline Measures & $\begin{array}{l}\text { - heart rate } \\
\text { - activity and sleep } \\
\text { - activity type } \\
\text { - calories burned } \\
\text { - body composition } \\
\text { - stress levels }\end{array}$ & $\begin{array}{l}\text { - heart rate } \\
\text { - activity and sleep } \\
\text { - activity type } \\
\text { - calories burned } \\
\text { - auto-exercise recognition } \\
\text { - multi-sport tracking }\end{array}$ & $\begin{array}{l}\text { - heart rate } \\
\text { - activity and sleep } \\
\text { - activity type } \\
\text { - calories burned }\end{array}$ \\
\hline Display & - Touch screen & - OLED display & - \\
\hline Mobile App & iOS / Android & iOS / Android / Windows & $\mathrm{iOS}$ \\
\hline Battery Life & $\begin{array}{l}\text { - } 5 \text { days } \\
\text { - charges in } 60 \text { mins }\end{array}$ & $\begin{array}{l}\text { - } 5 \text { days } \\
\text { - charges in } 120 \text { mins }\end{array}$ & $\begin{array}{l}\text { - } 3 \text { days } \\
\text { - charges in } 90 \text { mins }\end{array}$ \\
\hline Price & $145 €(180 \$)$ & $160 €$ & $160 €(200 \$)$ \\
\hline
\end{tabular}

\section{Evaluation criteria}

According to the papers described above, the evaluation criteria were established in Section 3.1 and the criteria weight in Section 3.2.

\subsection{Establishing the evaluation criteria}

The evaluation criteria of wearable activity-fitness trackers can be performed according to different aspects $[3,4]$. Three aspects of evaluation criteria are considered in this paper: social, economic and technological. In order to evaluate the alternatives, the following 11 criteria were established:

A. Manufacturing cost: refers to the cost of production and implementation.

B. Product utility: refers to the relevance of alternative wearable trackers.

C. Amount of information: based on the amount of data provided by these devices.

D. Accuracy of information: based on the accuracy of data provided by these devices.

E. Data monitoring: refers to the usability of data recorded by alternative trackers. 
F. Customer perception: refers to the customers' perception towards wearable devices.

G. Ease of use: usability is the criterion.

H. Comfort level: this criterion refers to the sense of comfort.

I. Design: the design of alternative activity-fitness trackers is the criterion.

J. Battery lifetime: based on battery lifetime of alternative activity-fitness trackers.

K. Waterproof: refers to the waterproof feature.

\subsection{Assessment of criteria weights}

In the assessment of criteria weights, the relevant decision-making experts from academic institutes and research organization, assessed the relative importance for each of the criteria. They defined the relationship between every pair of criteria by comparing every criterion against the others and filled a quadratic matrix that presents how these criteria relate to each other. When comparing two criteria the decision-making experts faced three possible situations: when a criterion on a row is compared with a criterion on a column, if it is more important, the value ' 1 ' is assigned, if it is equally important, the value ' 0,5 ' is assigned, and if it is less important, the value ' 0 ' is assigned. After the relationship matrix is complete, FRISCO formula (1) is applied to rank the criteria [4]:

$$
\gamma_{\mathrm{i}}=\frac{\mathrm{p}+\Delta \mathrm{p}+\mathrm{m}+0,5}{-\Delta \mathrm{p}^{\prime}+\mathrm{N}_{\mathrm{crt} / 2}}
$$

The weights $\left(\gamma_{i}\right)$ for each criterion, calculated using the FRISCO formula (1), are shown in the Table 2. This data shows that the Product utility is the most important factor in evaluating the alternative activity-fitness trackers, followed by Accuracy of Information.

Table 2. Criteria Weights, Calculated with FRISCO Formula.

\begin{tabular}{cccccccccccc|c|c|c|} 
& $\mathrm{A}$ & $\mathrm{B}$ & $\mathrm{C}$ & $\mathrm{D}$ & $\mathrm{E}$ & $\mathrm{F}$ & $\mathrm{G}$ & $\mathrm{H}$ & $\mathrm{I}$ & $\mathrm{J}$ & $\mathrm{K}$ & Score & Rank & $\gamma_{\mathbf{i}}$ \\
$\mathrm{A}$ & 0,5 & 0,5 & 0 & 0 & 0,5 & 0 & 1 & 1 & 0 & 1 & 1 & 5,5 & 6,5 & 1,69 \\
$\mathrm{~B}$ & 0,5 & 0,5 & 1 & 0,5 & 0,5 & 0 & 1 & 1 & 1 & 1 & 1 & 8 & 1 & 4,45 \\
$\mathrm{C}$ & 1 & 0 & 0,5 & 0 & 0,5 & 1 & 1 & 0 & 1 & 1 & 0,5 & 6,5 & 4 & 2,64 \\
$\mathrm{D}$ & 1 & 0,5 & 1 & 0,5 & 1 & 0,5 & 1 & 0 & 1 & 0,5 & 0,5 & 7,5 & 2 & 3,75 \\
$\mathrm{E}$ & 0,5 & 0,5 & 0,5 & 0 & 0,5 & 0 & 0,5 & 0 & 0 & 1 & 0 & 3,5 & 10 & 0,65 \\
$\mathrm{~F}$ & 1 & 1 & 0 & 0,5 & 1 & 0,5 & 0,5 & 0,5 & 0,5 & 0,5 & 0 & 6 & 5 & 2,2 \\
$\mathrm{G}$ & 0 & 0 & 0 & 0 & 0,5 & 0,5 & 0,5 & 0,5 & 0 & 0 & 0 & 2 & 11 & 0,22 \\
$\mathrm{H}$ & 0 & 0 & 1 & 1 & 1 & 0,5 & 0,5 & 0,5 & 0,5 & 1 & 1 & 7 & 3 & 3,15 \\
$\mathrm{I}$ & 1 & 0 & 0 & 0 & 1 & 0,5 & 1 & 0,5 & 0,5 & 0,5 & 0,5 & 5,5 & 6,5 & 1,69 \\
$\mathrm{~J}$ & 0 & 0 & 0 & 0,5 & 0 & 0,5 & 1 & 0 & 0,5 & 0,5 & 1 & 4 & 9 & 0,89 \\
$\mathrm{~K}$ & 0 & 0 & 0,5 & 0,5 & 1 & 1 & 1 & 0 & 0,5 & 0 & 0,5 & 5 & 8 & 1,35
\end{tabular}

\section{Evaluation of the alternatives}

In this stage of MCA, the alternatives are awarded with grades $\left(\mathrm{N}_{\mathrm{i}}\right)$ from 1 to $10 . \mathrm{N}_{\mathrm{i}}$ is the performance value given by experts to the alternatives, in relation to each criterion. Table 3 shows the grades awarded to the three alternatives [4].

In order to establish the final ranking of the three alternatives, the performance index $\left(\mathrm{F}_{\mathrm{i}}\right)$ is determined by the relation $(2)$ :

$$
\mathrm{F}_{\mathrm{i}}=\mathrm{N}_{\mathrm{i}} \times \gamma_{\mathrm{i}}
$$

Based on the performance index $\left(\mathrm{F}_{\mathrm{i}}\right)$ values, the final score was calculated for each alternative. The obtained ranking list is presented Table 4. 
Table 3. Grades Awarded to the Three Alternatives in Relation to Each Criterion.

\begin{tabular}{l|c|c|c} 
Criterion & InBody WATCH & Fitbit Charge 2 & Motiv Ring \\
\hline A - Manufacturing Cost & $\mathrm{N}_{\mathrm{i}}$ & $\mathrm{N}_{\mathrm{i}}$ & $\mathrm{N}_{\mathrm{i}}$ \\
B - Product utility & 10 & 9 & 9 \\
C - Amount of Information & 8 & 7 & 9 \\
D - Accuracy of Information & 10 & 9 & 7 \\
E - Data monitoring & 10 & 10 & 9 \\
F - Customer Perception & 10 & 9 & 8 \\
G - Ease of use & 8 & 7 & 10 \\
H - Comfort Level & 10 & 10 & 10 \\
I - Design & 7 & 7 & 10 \\
J - Battery Lifetime & 7 & 9 & 7 \\
K - Waterproof & 10 & 6 & 10
\end{tabular}

Table 4. Multi-criteria Ranking Results.

\begin{tabular}{|c|c|c|c|c|c|c|c|}
\hline \multirow{2}{*}{ Criterion } & \multirow{2}{*}{$\gamma_{\mathrm{i}}$} & \multicolumn{2}{|c|}{ InBody WATCH } & \multicolumn{2}{|c|}{ Fitbit Charge 2} & \multicolumn{2}{|c|}{ Motiv Ring } \\
\hline & & $\mathrm{N}_{\mathrm{i}}$ & $\mathrm{F}_{\mathrm{i}}$ & $\mathrm{N}_{\mathrm{i}}$ & $\mathrm{F}_{\mathrm{i}}$ & $\mathrm{N}_{\mathrm{i}}$ & $\mathrm{F}_{\mathrm{i}}$ \\
\hline Manufacturing Cost & 1,69 & 10 & 16,9 & 9 & 15,21 & 9 & 15,21 \\
\hline Product utility & 4,45 & 8 & 35,6 & 7 & 31,15 & 9 & 40,05 \\
\hline Amount of Information & 2,64 & 10 & 26,4 & 9 & 23,76 & 7 & 18,48 \\
\hline Accuracy of Information & 3,75 & 10 & 37,5 & 10 & 37,5 & 9 & 33,75 \\
\hline Data monitoring & 0,65 & 10 & 6,5 & 9 & 5,85 & 8 & 5,2 \\
\hline Customer Perception & 2,2 & 8 & 17,6 & 7 & 15,4 & 10 & 22 \\
\hline Ease of use & 0,22 & 10 & 2,2 & 10 & 2,2 & 10 & 2,2 \\
\hline Comfort Level & 3,15 & 7 & 22,05 & 7 & 22,05 & 10 & 31,5 \\
\hline Design & 1,69 & 7 & 11,83 & 6 & 10,14 & 10 & 16,9 \\
\hline Battery Lifetime & 0,89 & 10 & 8,9 & 9 & 8,01 & 7 & 6,23 \\
\hline Waterproof & 1,35 & 1 & 1,35 & 6 & 8,1 & 10 & 13,5 \\
\hline \multicolumn{2}{|l|}{ Final Score } & - & 186,83 & - & 179,37 & - & 205,02 \\
\hline \multicolumn{2}{|l|}{ Final Ranking } & \multicolumn{2}{|c|}{2} & \multicolumn{2}{|c|}{3} & \multicolumn{2}{|c|}{1} \\
\hline
\end{tabular}

\section{Results}

For this case study, the set of alternatives is specified in Section 2, the set of decision criteria is specified in Section 3, and the performances obtained for each alternative according to the decision criteria are specified in Section 4. According to the data presented in Table 4, the optimal alternative proposed as a solution is the best ranked alternative, and in this case, it is alternative no. 3 - Motiv Ring (final score 205,02).

\section{Smart jewellery - 3D models for rapid prototyping}

The result of MCA is that the smart jewellery are more suitable at present as activity-fitness tracking devices in order to improve the healthcare system. In this section, following the result of MCA, two 3D concept models of jewellery are presented for rapid prototyping: a ring (Figure 1) and a bracelet (Figure 2). The CAD files were created in SolidWorks. Shaped in a way to make better contact with the skin, these devices fit seamlessly into everyday life, being beautiful designed, comfortable, lightweight and user-friendly. Conceived for rapid prototyping, these two models can be used to create new tracking devices and to ensure the users' needs. Already used in jewellery industry, 3D printing technique simplifies the manufacturing process by providing the most suitable solution for customer's needs and expectations. Once created the jewellery, the customer can buy the 
optical heart rate sensor and place it into the flat section. This way, a simple jewellery becomes a smart tracker.

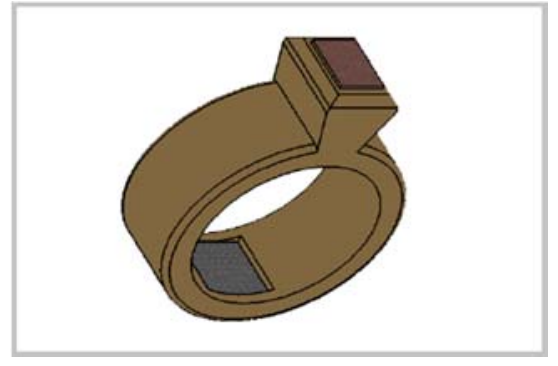

Fig. 1. Ring Concept Model for 3D Printing.

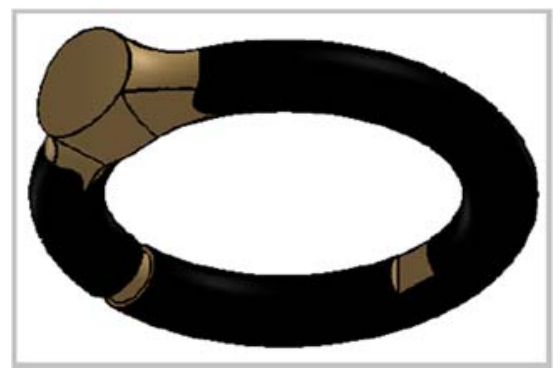

Fig. 2. Bracelet Concept Model for 3D Printing.

\section{Conclusions}

Until now, the customers have made sacrifices being forced to choose between style and comfort and a few hours of data insights and metrics. Thanks to the microtechnology developed by Motiv Inc and to the 3D printing manufacturing for commercial purpose, those who wear an activity-fitness tracker can now buy a beautiful designed device. Research firm Endeavor Partners indicates that, nowadays, to achieve long-term engagement, tracking devices can't simply provide data; they need to provide clear insights that inspire action [2]. To help users staying motivated, researchers must create new trackers that can be worn all the time. The result of MCA indicates that the most preferred fitness tracker is a discreet device, with basic needs, which can be worn on all occasions: a small, durable, waterproof device, allowing to effortlessly measure activity and sleep, and not to interfere with daily life.

\section{References}

1. B. Abaidoo, B.T. Larweh, Online J Public Health Inform 6, 2 (2014)

2. Revolutionizing the Wearable Experience, Motiv Ring is the First Activity + Sleep Tracker That Fits Seamlessly into Everyday Life, - http://www.prnewswire.com/newsreleases/revolutionizing-the-wearable-experience-motiv-ring-is-the-first-activity-sleep-tracker-that-fits-seamlessly-into-everyday-life-300384480.html; Accessing date: 30.01.2018

3. Multi-criteria analysis: a manual, https://www.gov.uk/government/uploads/system/uploads/attachment_data/file/7612/11 32618.pdf; Accessing date: 10.01.2018

4. Ş. Bobancu, Creativity and inventics (in Romanian), http://www.unitbv.ro/Portals/31/Scoala\%20DOctorala/Curs\%20CI\%20\%20Sectiunea\%201\%20si\%202.pdf; Accessing date: 14.01.2018

5. J.G. Conley, H.L. Marcus, J Manuf Sci Eng 119 (1997)

6. CES 2017 Honorees, - http://www.ces.tech/Events-Experiences/Innovation-AwardsProgram/Honorees.aspx; Accessing date: 14.01.2018

7. InBody, - http://www.bodykey.my/en_us/products/inbodyband.html; Accessing date: 14.01.2018

8. FitBit, - https://www.fitbit.com/charge2; Accessing date: 14.01.2018

9. Motiv Ring, - https://mymotiv.com/; Accessing date: 14.01.2018 